Artículo científico

Volumen 33(1): 43350, 2022

e-ISSN 2215-3608, doi:10.15517/am.v33i2.43350

https://revistas.ucr.ac.cr/index.php/agromeso/index

\title{
Parasitismo de huevos de lepidópteros plagas (Insecta: Lepidoptera) por Trichogramma pretiosum (Hymenoptera: Trichogrammatidae) en tomate (Solanum lycopersicum) $^{1}$
}

\section{Egg parasitism of Lepidoptera pests (Insecta: Lepidoptera) by Trichogramma pretiosum (Hymenoptera: Trichogrammatidae) in tomato (Solanum lycopersicum)}

\author{
Jorge Salas ${ }^{2}$, Francisco Ferrer-Wurst ${ }^{3}$
}

1 Recepción: 10 de marzo, 2021. Aceptación: 3 de septiembre, 2021. Este trabajo formó parte del proyecto de investigación "Manejo Integrado de Plagas en Hortalizas en el estado Lara", financiado por Convenio Instituto Nacional de Investigaciones (INIA) - Gobernación del Estado Lara, Venezuela.

2 Instituto Nacional de Investigaciones Agrícolas (INIA-Lara), Barquisimeto, Venezuela. salasjl@hotmail.com (http://orcid.org/0000-00028379-5034).

3 Corporación de Desarrollo Endógeno y Economía Social. Gobernación del Estado Lara, Venezuela. donfranciscowurst@gmail.com (autor para correspondencia; http://orcid.org/0000-0001-7064-4379).

\section{Resumen}

Introducción. El perforador del fruto del tomate Neoleucinodes elegantalis (Guenée) (Lepidoptera: Pyralidae) y los minadores de la hoja Phthorimaea operculella (Zeller) y Tuta absoluta Meyrick (Lepidoptera: Gelechiidae), afectan la producción de tomate en diferentes áreas en Venezuela. El parasitismo de sus huevos por Trichogramma pretiosum Riley (Hymenoptera: Trichogrammatidae) es una valiosa alternativa de control de bajo costo y sustentable que combinado con otras formas de control sería un excelente componente del Manejo Integrado de Plagas. Objetivo. Evaluar el parasitismo ejercido por T. pretiosum sobre las poblaciones de plagas lepidópteras en tomate. Materiales y métodos. La evaluación se realizó en todas las siembras entre junio y agosto de 2017, desde la tercera semana después del trasplante hasta dos semanas después de la última floración, en las localidades El Pueblito, Los Ejidos del Hato y El Vigiadero, municipio Jiménez, estado Lara, Venezuela. Se liberaron los adultos del parasitoide y posteriormente, se recolectaron los foliolos para evaluar el parasitismo de los huevos de las especies minadoras. Al iniciarse la fructificación, se colectaron frutos pequeños y medianos incluyendo el cáliz y fueron cortados cuidadosamente del pedúnculo floral. En ambos casos se realizó el conteo de los huevos. Resultados. En todas las evaluaciones, se registró un parasitismo que varió en 43 y $53 \%$ para $N$. elegantalis y entre 16 y $48 \%$ para las especies minadoras de las hojas. En el laboratorio, el parasitismo para P. operculella y T. absoluta varió entre 88 y $84 \%$ cuando los huevos estuvieron separados, al estar juntos se observó un mayor parasitismo de huevos de P. operculella (76\%) versus T. absoluta (44 $\%)$. Conclusiones. $T$. pretiosum ejerció un parasitismo significativo sobre los huevos de las tres especies lepidópteras estudiadas en siembras de tomate en Venezuela.

Palabras claves: Neoleucinodes, Phthorimaea, Tuta, Trichogramma, manejo integrado de plagas, hortalizas. 


\begin{abstract}
Introduction. The tomato fruit borer Neoleucinodes elegantalis (Guenée) (Lepidoptera: Pyralidae), and the leafminers Phthorimaea operculella (Zeller) and Tuta absoluta Meyrick (Lepidoptera: Gelechiidae), affect tomato production in different areas in Venezuela. The parasitism of their eggs by Trichogramma pretiosum Riley (Hymenoptera: Trichogrammatidae) is a valuable low-cost and sustainable control alternative that combined with other forms of control would be an excellent component of Integrated Pest Management. Objective. To evaluate the parasitism exerted by T. pretiosum on the lepidopteran pest populations in tomato. Materials and methods. The evaluation was carried out in all sowings between June and August 2017, from the third week after the transplant until two weeks after the last flowering, in the localities of El Pueblito, Los Ejidos del Hato, and El Vigiadero, Jiménez municipality, Lara state, Venezuela. Parasitoid adults were released and subsequently leaflets were collected to evaluate the parasitism of the eggs of the leafminer species. At the onset of fruiting, small and medium-sized fruits including the calyx were collected and carefully cut from the floral peduncle. In both cases, eggs were counted. Results. In all evaluations, a parasitism varied between 43 and $53 \%$ for N. elegantalis, and between 16 and $48 \%$ for leafminer species was recorded. In the laboratory, the parasitism for $P$. operculella and $T$. absoluta ranged between 88 and $84 \%$, when eggs were separated, when eggs were together, a higher parasitism of $P$. operculella eggs (76\%) versus T. absoluta (44\%) was observed. Conclusions. T. pretiosum exerted a significant parasitism on the eggs of the three-lepidopteran species in tomato crops in Venezuela.
\end{abstract}

Keywords: Neoleucinodes, Phthorimaea, Tuta, Trichogramma, integrated pest management, vegetable crops.

\title{
Introducción
}

El complejo de insectos plaga conformado por el perforador del fruto Neoleucinodes elegantalis (Guenee) (Lepidoptera: Pyralidae) y los minadores de la hoja Phthorimaea operculella (Zeller) y Tuta absoluta Meyrick (Lepidoptera: Gelechiidae), representa una gran amenaza para la producción de tomate (Solanum lycopersicum) en áreas bajas y altas del estado Lara y en el resto de Venezuela. N. elegantalis destruye total y exclusivamente al fruto, mientras que P. operculella y T. absoluta atacan al follaje y a los frutos. El control de estas plagas en las áreas antes mencionadas, generalmente se ha enfocado en la utilización de insecticidas organosintéticos, los cuales no ejercen un control satisfactorio, en la mayoría de los casos el nivel de acción es muy bajo y su uso no es sustentable en el tiempo. Esta situación sugiere el uso combinado de otras alternativas de control, dentro del enfoque conocido como manejo integrado de plagas (MIP). Dentro de las técnicas del MIP, el control biológico es una de ellas.

Los parasitoides de huevos del género Trichogramma, han sido ampliamente estudiados y utilizados en muchos países como agentes de control biológico (Fuentes et al., 2012). El parasitoide de huevos Trichogramma spp. (Hymenoptera: Trichogrammatidae), ha sido reportado mundialmente como un importante controlador biológico de huevos de diferentes especies plagas del orden Lepidoptera. T. pretiosum ha sido señalada como un importante controlador de Spodoptera cosmioides Walker a nivel de laboratorio a diferentes temperaturas (Cabezas et al., 2013). Esta misma especie ha causado un parasitismo entre 60-100\% de Alabama argillacea Hubner y Heliothis virescens (F.) en siembras de algodón en Brasil (Fernandes et al., 1999). T. pretiosum se reportó por Massaroli et al. (2014) como un parasitoide de natural ocurrencia en siembras de soya en la región de Mato Grosso, Brasil, el cual parasita huevos de especies de los géneros Spodoptera y Helicoverpa. Recientemente, T. pretiosum liberado por métodos terrestre y aéreo (dron) contra plagas lepidópteras en siembras de soya en Uruguay, alcanzaron los mejores resultados por debajo de los umbrales de intervención sanitaria (Basso et al., 2020). A nivel de laboratorio, Laurentis et al. (2019), encontraron a T. pretiosum como una útil herramienta para el manejo integrado del insecto plaga polífago Heliothis armígera en Brasil. 
La polilla suramericana del tomate (Tuta absoluta), en condición de "plaga invasora" se reportó por primera vez en 2006 en Europa, en la provincia de Castellón ubicada al este de España. T. absoluta se ha expandido con gran rapidez en los últimos tres años (2007-2009) por casi todos los países del Mediterráneo, excepto el extremo oriental, así como por muchos países centroeuropeos (Ferrán-García \& Vercher, 2010). Recientemente, Mukwa et al. (2021) reportaron por primera vez la presencia y el daño causado por $T$. absoluta en siembras de tomate localizadas en el área de Nsele en la República Democrática del Congo (RDC), al sureste de África. La presencia de esta plaga invasora en Europa, África y el Oriente Medio, ha conllevado al desarrollo de programas de investigación para su manejo integrado y el uso del control biológico a través de parasitoides y depredadores; entre ellos, parasitoides de huevos del género Trichogramma con especies nativas.

Trichogramma pretiosum ha sido reportado por parasitar huevos de T. absoluta en genotipos de tomate conteniendo una sustancia que les confiere resistencia a esa plaga, se observó el mayor parasitismo en el genotipo menos resistente (Rodrigues Gonçalves-Gervásio et al., 2000). Al evaluar el parasitismo de T. pretiosum junto a otras tres especies, con base en la densidad de liberación y la distancia desde el punto de liberación en invernaderos, T. pretiosum tuvo el menor parasitismo ejercido (Sarhan et al., 2015). En África, después de la aparición y establecimiento de T. absoluta en diversos cultivos y preferentemente en cultivos solanáceos, se ha planteado entre las estrategias de control el uso de depredadores y hasta trece diferentes especies de la avispita parasitoide del género Trichogramma, incluyendo a T. pretiosum (Tarusikirwa et al., 2020).

Otras especies de Trichogramma diferentes a T. pretiosum, han sido citadas como controladores de T. absoluta, con diferentes valores de parasitismo y considerados como exitosos, en algunos casos combinados con otras medidas de control y en diferentes condiciones y localidades. T. achaeae ha sido señalado como un muy exitoso controlador de T. absoluta con parasitismo entre 87-90 \% (Cabello et al., 2009). Se reportó a T. cacoeciae como un altamente eficiente controlador de huevos de T. absoluta a nivel de laboratorio e invernaderos en el suroeste de Túnez, África del Norte (Zouba \& Mahjoubi, 2010). Años más tarde, se evaluó nuevamente el parasitismo de huevos de T. absoluta ejercido T. achaeae y otra especie T. urquijoi, combinadas o no con el depredador Nesidiocoris tenuis (Hemiptera: Miridae), se encontró que T. achaeae controló mejor las poblaciones que la otra especie y además se observó una competencia interespecífica del depredador en ambas especies de Trichogramma (Cabello et al., 2012). Otras especies de Trichogramma diferentes a T. pretiosum, han mostrado un bajo parasitismo en huevos de T. absoluta en siembras de tomate en Irán, mientras que T. evanescens fue la que infringió el mayor parasitismo (26,82 \%) (Chamaani \& Poorjavad, 2020). Nueve especies europeas de Trichogrammma se evaluaron a nivel de laboratorio en la capacidad de parasitar, en comparación con T. achaeae, especie usada y disponible comercialmente, encontrándose que T. nerudai, T. pintoi y T. cacoeciae lograron un nivel de parasitismo similar a T. achaeae (Shäfer \& Herz, 2020). En invernaderos, se evaluó el control biológico de huevos de T. absoluta por T. brassicae unido a otra medida de control de la plaga, en este caso una variedad resistente al ataque de la plaga, en ambos controles no solo redujo el daño causado, además disminuyeron el número de aplicaciones de insecticidas (Saeidi \& Raeesi, 2021).

Publicaciones referentes al parasitismo de huevos de $P$. operculella en siembras de cultivos hospederos son limitadas. Se han reportado a adultos de T. pretiosum colectados de huevos de esta plaga presentes en tubérculos de papa almacenados en galpones en las zonas altas del estado Lara, Venezuela (Morales et al., 2007). Recientemente, se evaluó el potencial biológico de las especies Trichogramma achaeae y T. cacoeciae como agentes de control de P. operculella en España (Gallego et al., 2020). Ambas especies parecen ser buenos candidatos, ya que ejercieron $92,78 \%$ y $70,88 \%$ de parasitismo, respectivamente.

En cuanto al parasitismo de huevos de N. elegantalis, Blackmer et al. (2001) observaron y colectaron al parasitoide de huevos T. pretiosum al final de la época de siembra de tomate en San José de Uba, Brasil, en donde, conforme fue aumentando el número de huevos de $N$. elegantalis, se redujo el uso de insecticidas y el nivel de parasitismo aumentó de 2,4 a 28,7\% durante la últimas evaluaciones.

Agron. Mesoam. 33(1): 43350, 2022

ISSN 2215-3608 doi:10.15517/am.v33i2.43350 
Se reportó un amplio grupo de enemigos naturales de Neoleucinodes elegantalis en Colombia, los cuales se encontraron en siembras afectando sus huevos, larvas y pupas, pero solo se informó de una especie no determinada del parasitoide de huevos Trichogramma en tomate (Díaz \& Brochero, 2012). Se indicó a T. pretiosum como un promisorio agente de control biológico de esta plaga, después de liberarlo en siembras de tomate (Moura de Oliveira et al., 2017). También se evaluaron tres métodos de control sobre poblaciones de N. elegantalis en parcelas experimentales de tomate establecidas en el sector Los Ríos, municipio Seboruco, estado Táchira, Venezuela; estudio en el cual el tratamiento con el parasitoide de huevos T. pretiosum, registro la menor infestación (12,6\%), el mejor rendimiento y la mayor relación beneficio-costo (B/C), en relación a los otros métodos de control (Silva Alarcón, 2020).

El objetivo de este trabajo fue evaluar el parasitismo ejercido por T. pretiosum sobre las poblaciones de plagas lepidópteras en tomate.

\section{Materiales y métodos}

Las evaluaciones del parasitismo ejercido por la avispita $T$. pretiosum sobre los huevos de $N$. elegantalis (perforador del fruto), y en P. operculella y T. absoluta (minadores de la hoja) se realizaron entre junio y agosto de 2017, en dos lotes por localidad de $2500 \mathrm{~m}^{2}$ (50x50m) sembrados de tomate (Solanum lycopersicum), variedad Río Grande (Semillas Magna), tipo pera, establecidos en diferentes épocas en las localidades El Pueblito, Los Ejidos del Hato y El Vigiadero, cercanas a Quíbor, capital del municipio Jiménez, estado Lara, Venezuela, ubicadas a $9^{\circ} 53^{\prime} \mathrm{N}$ y $69^{\circ} 39^{\prime} \mathrm{O}, 680$ m.s.n.m. y con una temperatura y precipitación promedio de $30^{\circ} \mathrm{C}$ y $575 \mathrm{~mm}$, respectivamente.

Las avispitas se obtuvieron del Laboratorio de la Asociación Nacional de Cultivadores de Algodón C. A. (ANCA C.A.), dispensadas en cartones con huevos de Sitotroga cerealella Olivier (Lepidoptera: Gelechiidae), previamente parasitados.

Los cartones de 50 o 100 pulg$^{2}$ de huevos de T. pretiosum prontos a emerger, se colocaron en frascos de plástico boca ancha de un galón, los cuales se taparon con una pieza de tela gruesa de color negro y se sujetaron con una banda de goma gruesa. Una vez que se observó el inicio de la emergencia de los parasitoides, se esperó a que hubiera un $90 \%$ de emergencia, ya que la pared del frasco se oscurecía y se podían ver las avispitas en movimiento. Al día siguiente, se realizó la liberación de las avispitas en la tarde de 4-5 pm, para evitar las altas temperaturas. Las liberaciones se hicieron recorriendo al inicio el primer hilo de siembra del lote y luego se recorrió otro hilo de siembra cada diez hilos, o sea el hilo \# 11 y así sucesivamente. El recorrido se hizo con el frasco abierto, el cual se acercaba a la planta; también se introdujo una hoja de papel facial blanco dentro del frasco, los parasitoides rápidamente se posaron en gran número y ese papel se colocó en el centro de una planta entre el follaje. Una vez que se recorrió la siembra bajo ese esquema, si aún quedaron avispitas dentro del frasco, el lote se recorrió en reversa en la misma manera antes descrita. Cuando quedaron pocas avispitas en el frasco, se colocaron trozos de cartón de 4 pulg $^{2}$, al azar y bien distribuidos, y se fijaron en una planta dentro del follaje con un trozo de cordel o un clip, con el fin de que aquellas avispitas que no habían emergido lo hicieran posteriormente.

Se realizaron entre siete y nueve liberaciones del parasitoide en las tres localidades. En los dos lotes de siembra de cada localidad se hicieron liberaciones desde la tercera semana después del trasplante hasta el final del cultivo. En la localidad El Pueblito, se liberaron 900 pulg$^{2}$ en el primer lote y 1300 pulg$^{2}$ en el segundo. En Los Ejidos del Hato, se procedió de la misma manera, pero en el primer lote se liberaron 850 pulg$^{2}$ y en el segundo 1250 pulg$^{2}$, mientras que, en El Vigiadero, se liberaron 1200 pulg$^{2}$ en ambos lotes.

Para el muestreo de huevos de $N$. elegantalis, P. operculella y T. absoluta, se siguió la metodología de muestreo aleatorio simple por separado para los minadores de la hoja y el perforador del fruto. Cada localidad dividida en dos lotes de siembra, fue a su vez subdividida en cuatro sub-lotes o cuadrículas de $(25 \times 25 \mathrm{~m})$ para un área de $625 \mathrm{~m}^{2}$. Para 
los minadores de la hoja (P. operculella y T. absoluta), en cada sub-lote se tomaron 50 foliolos al azar bien distribuidos en las diferentes plantas y aún dentro de los tres tercios de la misma, para un total de 200 foliolos por lote y por conteo, entre cuatro y cinco días después de la liberación. Esos foliolos se colocaron en bolsas plásticas con una pieza de papel absorbente, debidamente identificadas y se trasladaron en una cava refrigerada al laboratorio para el conteo de huevos parasitados o no. Para el conteo de los huevos en los frutos, se tomaron 25 por cada sub-lote para un total de 100 frutos por lote, incluyendo el cáliz, los cuales se cortaron cuidadosamente con una tijera de podar en la parte superior del pedúnculo floral, ya que mayormente N. elegantalis coloca sus huevos en el cáliz (Salas et al., 1991), mientras que $P$. operculella y T. absoluta lo hacen en ambas estructuras, pero preferiblemente sobre el fruto. Esos frutos se colocaron cautelosamente en cartones de colocar huevos de gallina, los cuales a su vez, se pusieron en bolsas plásticas con una pieza de papel absorbente, debidamente identificadas y se trasladaron en una cava refrigerada al laboratorio para el conteo de huevos parasitados o no, en ambos casos con un microscopio estereoscópico.

Debido a que los huevos de ambas especies minadoras P. operculella y T. absoluta lucen muy similares, paralelamente al trabajo de campo y para determinar cuáles huevos de las dos especies eran parasitados en campo, se establecieron, en el laboratorio, crías por separado de ambas especies. Una vez obtenidos los huevos de cada una, se colocaron diez placas de Petri con diez huevos de cada especie para observar si eran parasitados. Para evaluar el parasitismo en ambas especies por separado, se colocaron al azar diez placas de Petri con cinco huevos de cada especie debidamente identificados (cinco de P. operculella y cinco de T. absoluta), y se contó el número de huevos parasitados o no. En ambas situaciones se introdujeron diez avispitas hembras recién emergidas por cada placa de Petri, bajo condiciones de temperatura de $20-22{ }^{\circ} \mathrm{C}$, una humedad relativa del $65 \%$ y una iluminación de 12:12 h. Los datos encontrados, se expresaron en porcentajes y se registraron en planillas diseñadas para tal fin. Se realizó un análisis de varianza no paramétrico para los factores localidad y tipo de plaga, en donde las diferencias de las medias se discriminaron por la prueba de Kruskal Wallis y prueba de t. Todos los análisis se realizaron con el Programa InfoStat versión 2020 (Di Rienzo et al., 2020).

\section{Resultados}

Hubo parasitismo ejercido por T. pretiosum sobre las poblaciones de huevos de N. elegantalis, P. operculella y T. absoluta, en siembras de tomate establecidas en dos épocas y en tres localidades de Quibor.

Las localidades mostraron un comportamiento similar del parasitismo ejercido por T. pretiosum sobre los huevos del perforador del fruto Neoleucinodes elegantalis, así como sobre los minadores de la hoja. Sin embargo, el análisis mostró diferencias significativas entre el porcentaje de parasitismo ejercido por T. pretiosum sobre el perforador del fruto y los minadores de la hoja con valores de 48,45\% y 28,68\%, respectivamente (Cuadro 1).

En la localidad El Pueblito, el parasitismo de huevos de N. elegantalis en el primer lote de siembra alcanzó un 50,20\%, mientras que en la segunda siembra fue 51,30\%. En Los Ejidos del Hato fue similar en los dos lotes de siembra, con 48,20 y 52,70 \%, respectivamente. En la localidad El Vigiadero el parasitismo fue ligeramente menor, 42,72 y 45,60\%. Respecto al parasitismo de huevos de las especies de minadores de la hoja del tomate $P$. operculella y T. absoluta, en El Pueblito, el parasitismo observado fue $21,20 \%$ en la primera siembra y $24,80 \%$ en la segunda, mientras que en Los Ejidos del Hato el parasitismo observado fue casi el doble, 41,80 \% y 47,64 \%, respectivamente. En El Vigiadero, se obtuvo el menor parasitismo con 16,00 y 20,70 \% (Cuadro 2).

En el laboratorio, al observar los huevos de cada especie minadora de la hoja P. operculella y T. absoluta, se encontró que los huevos de ambas especies presentaron un parasitismo muy similar, cuando los huevos de cada especie estuvieron separados, con valores de 88 y $84 \%$, respectivamente. Sin embargo, se observó un parasitismo $32 \%$ mayor en favor de P. operculella sobre T. absoluta, cuando estuvieron juntos (Cuadro 3). Estos porcentajes estuvieron dentro los rangos aceptados de la distribución binomial a un nivel de $\mathrm{p}=95 \%$.

Agron. Mesoam. 33(1): 43350, 2022

ISSN 2215-3608 doi:10.15517/am.v33i2.43350 
Cuadro 1. Promedio del parasitismo de huevos de N. elegantalis y los minadores de la hoja (Phthorimaea operculella \& Tuta absoluta) discriminados por la prueba de Kruskal Wallis en siembras de tomate (Solanum lycopersicum), en tres localidades en Quibor, municipio Jiménez, estado Lara, Venezuela. 2017.

Table 1. Mean eggs parasitism of N. elegantalis and tomato leafminers (Phthorimaea operculella \& Tuta absoluta) discriminated by the Kruskal Wallis test in tomato (Solanum lycopersicum) sowings in three localities in Quibor, Jiménez municipality, Lara state, Venezuela. 2017.

\begin{tabular}{lcccccc}
\hline & \multicolumn{3}{c}{$\begin{array}{c}\text { Perforador del fruto } \\
\text { Neoleucinodes elegantalis }\end{array}$} & \multicolumn{2}{c}{$\begin{array}{c}\text { Minadores de la hoja } \\
\text { Phthorimaea operculella \& Tuta absoluta }\end{array}$} \\
\cline { 2 - 7 } Localidad & $\begin{array}{c}\text { Huevos } \\
\text { colectados }\end{array}$ & $\begin{array}{c}\text { Huevos } \\
\text { parasitados }\end{array}$ & \% Parasitismo & $\begin{array}{c}\text { Huevos } \\
\text { colectados }\end{array}$ & $\begin{array}{c}\text { Huevos } \\
\text { parasitados }\end{array}$ & \% Parasitismo \\
\hline El Pueblito & $297,00 \mathrm{a}$ & $151,00 \mathrm{a}$ & $50,75 \mathrm{a}$ & $235,50 \mathrm{a}$ & $55,00 \mathrm{a}$ & $22,99 \mathrm{a}$ \\
Los Ejidos & $491,00 \mathrm{a}$ & $249,50 \mathrm{a}$ & $50,45 \mathrm{a}$ & $224,00 \mathrm{a}$ & $101,00 \mathrm{a}$ & $44,70 \mathrm{a}$ \\
El Vigiadero & $273,00 \mathrm{a}$ & $121,50 \mathrm{a}$ & $44,16 \mathrm{a}$ & $194,00 \mathrm{a}$ & $36,50 \mathrm{a}$ & $18,37 \mathrm{a}$ \\
\hline Total & & $48,45 \mathrm{~A}$ & & & & $28,68 \mathrm{~B}$ \\
\hline
\end{tabular}

Medias con una letra común no son significativamente diferentes $(\mathrm{p}>0,05)$ para cada una de las variables. / Means with a common letter are not significantly different $(\mathrm{p}>0.05)$ for each of the variables.

Cuadro 2. Parasitismo de huevos de $N$. elegantalis y los minadores de la hoja (Phthorimaea operculella \& Tuta absoluta) por T. pretiosum en siembras de tomate (Solanum lycopersicum), en tres localidades en Quibor, municipio Jiménez, estado Lara, Venezuela. 2017.

Table 2. Egg parasitism of N. elegantalis and tomato leafminers (Phthorimaea operculella \& Tuta absoluta) by T. pretiosum in tomato (Solanum lycopersicum) sowings in three localities in Quibor, Jiménez municipality, Lara state, Venezuela. 2017.

\begin{tabular}{|c|c|c|c|}
\hline \multirow[b]{2}{*}{ Lotes } & \multirow[b]{2}{*}{ Localidad } & $\%$ & Parasitismo \\
\hline & & $\begin{array}{l}\text { Minadores de la hoja Phthorimaea } \\
\text { operculella \& Tuta absoluta }\end{array}$ & $\begin{array}{c}\text { Perforador del fruto } \\
\text { Neoleucinodes elegantalis }\end{array}$ \\
\hline \multirow[t]{3}{*}{1} & El Pueblito & 21,20 & 50,20 \\
\hline & Los Ejidos & 41,80 & 48,20 \\
\hline & El Vigiadero & 16,00 & 42,72 \\
\hline \multirow[t]{3}{*}{2} & El Pueblito & 24,80 & 51,30 \\
\hline & Los Ejidos & 47,64 & 52,70 \\
\hline & El Vigiadero & 20,70 & 45,60 \\
\hline $\mathrm{n}$ & & 6 & 6 \\
\hline Media & & 28,68 & 48,45 \\
\hline \multirow[t]{4}{*}{ Varianza } & & 165,09 & 14,06 \\
\hline & \multicolumn{3}{|c|}{ Media $(2)-\operatorname{Media}(1)=19,77 ; \operatorname{LI}(95)=43,14 ; \operatorname{LS}(95)=6,4$} \\
\hline & \multicolumn{3}{|c|}{ P Hom Var =0,0172 } \\
\hline & & $\mathrm{p}$-valor $=0,011$ & \\
\hline
\end{tabular}

*diferencia significativa. / *significant difference. 
Cuadro 3. Parasitismo de huevos de P. operculella y T. absoluta por T. pretiosum. Laboratorio del Instituto Nacional de Investigaciones Agrícolas (INIA). Venezuela. 2017.

Table 3. Egg parasitism of P. operculella and T. absoluta by T. pretiosum. Laboratory of Instituto Nacional de Investigaciones Agrícolas (INIA). Venezuela. 2017.

\begin{tabular}{lccc}
\hline Especies & \# huevos contados & \# huevos parasitados & $\begin{array}{c}\text { \% Parasitismo } \\
\text { Límites de confianza }(\mathbf{p}=\mathbf{9 5} \%) *\end{array}$ \\
P. operculella & 100 & 88 & $88(80-94)$ \\
T. absoluta & 100 & 84 & $84(75-91)$ \\
P. operculella + T. absoluta & $50+50$ & $38+22$ & $76(67-84)+44(34-54)$ \\
\hline
\end{tabular}

Se colocaron diez hembras de Trichogramma por cada especie. / Ten Trichogramma females were placed for each species. *Según distribución binomial al $95 \%$ de confianza. / * According to binomial distribution at $95 \%$ confidence.

\section{Discusión}

El parasitoide T. pretiosum fue capaz de parasitar los huevos de $N$. elegantalis en una mayor proporción numérica que los de las especies minadoras de la hoja T. absoluta y P. operculella, según las pruebas de Kruskal Wallis y de $t(p=0,05)$ (Di Rienzo et al., 2020).

Al comparar el parasitismo de huevos entre las especies minadoras P. operculella y T. absoluta en el laboratorio, ambas especies presentaron un alto parasitismo (88 y $84 \%$ ) al estar los huevos separados, mientras que, al estar juntos, el parasitismo fue mayor para P. operculella sobre T. absoluta (76 y $44 \%$ ). El parasitismo de huevos de estas tres especies lepidópteras que atacan el tomate y otros cultivos solanáceos, por T. pretiosum y otras especies de Trichogramma han sido referidos en variados cultivos y localidades a nivel mundial.

El parasitismo de huevos de T. absoluta por T. pretiosum en siembras de tomate es poco referido. Rodríguez Goncalves-Gervasio et al. (2000) lo reportaron combinado con otra estrategia de control en genotipos de tomate resistentes a esa plaga, observándose el mayor parasitismo en el genotipo menos resistente. Al evaluar el parasitismo de $T$. pretiosum junto a otras tres especies, teniendo en cuenta la densidad de liberación y la distancia desde el punto de liberación en invernaderos de tomate, Sarhan et al. (2015) encontraron que T. pretiosum ejerció el menor parasitismo. En África, después de la aparición y establecimiento de T. absoluta en diversos cultivos y, entre ellos los cultivos solanáceos, se ha planteado evaluar el parasitismo de hasta trece diferentes especies de la avispita parasitoide Trichogramma, incluyendo a T. pretiosum (Tarusikirwa et al., 2020).

Diferentes especies europeas de Trichogramma han sido evaluadas y algunas de ellas han resultado ser eficientes controladores de T. absoluta. Dentro de las especies controladoras reportadas se encuentran T. achaeae (Cabello et. al., 2009; 2012), T. bactrae (Riquelme Virgala \& Botto, 2010; Sarhan et al., 2015), T. cacoeciae y T. bourarachae (Mansour et al., 2019; Zouba \& Mahjoubi, 2010). Otras especies como T. nerudai, T. pintoi y T. cacoeciae lograron un nivel de parasitismo similar a $T$. achaeae, especie usada y disponible a nivel comercial (Shäfer \& Herz, 2020).

Al evaluar a $T$. evanescens, $T$. principium y $T$. brassicae en siembras de tomate en el campo, así como en jaulas en invernaderos, presentaron una capacidad parasitaria baja (menos del $8 \%$ ), aunque T. evanescens mostró la mayor capacidad de parasitismo (26,82 \%) (Chamaani \& Poorjavad, 2020). Pero se determinó que el control ejercido por $T$. brassicae unido a una variedad resistente, redujeron el daño causado y el número de aplicaciones de insecticidas (Saeidi \& Raeesi, 2021). Otras especies de Trichogramma no especificadas, son citadas como efectivas en la reducción poblacional de T. absoluta (Abdel-Razek et al., 2019; Gaffar et al., 2016). Similar a lo que sucede con el número de referencias del parasitismo de huevos de las otras dos plagas. Adultos de T. pretiosum emergieron 
de huevos de P.operculella en tubérculos de papa almacenados (Morales et al., 2007). Las dos especies parasitoides de huevos T. achaeae y T. cacoeciae usadas contra T. absoluta, son reportadas como exitosos agentes de control de P. operculella a nivel de laboratorio, registrándose una mortalidad total de huevos del 92,78 \% para T. achaeae y $70,88 \%$ para T. cacoeciae (Gallego et al., 2020).

T. pretiosum ha sido reportado por algunos autores por parasitar huevos de N. elegantalis. Lo colectaron al final de la época de siembra de tomate en San José de Uba, Brasil, cuando el número de huevos de $N$. elegantalis fue incrementándose y el uso de insecticidas reduciéndose con el consecuente aumento del nivel de parasitismo, el cual se incrementó a 28,7 \% (Blackmer et al., 2001). Se reportó un amplio grupo de enemigos naturales de N. elegantalis en Colombia, los cuales se encontraron en siembras afectando sus huevos, larvas y pupas (Díaz \& Brochero, 2012), pero solo se reportó una especie no determinada del parasitoide de huevos Trichogramma sp en tomate. Se indica que T. pretiosum se ha liberado con éxito en cultivos de tomate en Brasil, y por lo tanto, podría ser un agente de control biológico prometedor del barrenador de la fruta del tomate N. elegantalis (Moura de Oliveira et al., 2017). Tres métodos de control sobre poblaciones de $N$. elegantalis se evaluaron en parcelas experimentales de tomate establecidas en el sector Los Ríos, estado Táchira, Venezuela, y el tratamiento biológico a través del parasitoide de huevos $T$. pretiosum, registró la menor infestación, el mejor rendimiento y la mayor relación beneficio-costo (B/C) en relación con los otros métodos de control (Silva Alarcón, 2020).

El parasitoide de huevos T. pretiosum mostró una preferencia de parasitismo, entre fruto y hojas. Hubo una preferencia por huevos de $N$. elegantalis, seguido de P. operculella y T. absoluta, a nivel de siembra, cuando las tres especies ocurrieron en un mismo tiempo, al considerar que la permanencia de N. elegantalis en el cultivo fue menor en tiempo a partir de la floración, mientras que P. operculella y T. absoluta lo hicieron desde semillero a fructificación. Además, esa preferencia por huevos de $P$. operculella se observó en el laboratorio bajo condiciones controladas.

\section{Conclusiones}

T. pretiosum ejerció un parasitismo significativo sobre los huevos de $N$. elegantalis, en comparación con los huevos de P. operculella y T. absoluta, en siembras de tomate en las condiciones de las localidades evaluadas en el estado Lara, Venezuela.

En el laboratorio, T. pretiosum mostró preferencia por los huevos de P. operculella versus los de T. absoluta, cuando los huevos de ambas especies estuvieron juntos.

Se sugiere la validación de Trichogramma pretiosum en programas de manejo integrado de plagas en cultivos hortícolas que son atacados por estas especies plaga, ya que se presenta como una alternativa de control viable y sustentable.

\section{Referencias}

Abdel-Razek, A. S., Masry, S. H. D., Sadek, H. E., \& Gaafar, N. M. (2019). Efficacy of Trichogramma wasps for controlling tomato leaf miner Tuta absoluta. Archives of Phytopathology and Plant Protection, 52(5-6), 443-457. https://doi.or g/10.1080/03235408.2019.1634782

Basso, C., Chiaravalle, W., \& Maignet, P. (2020). Effectiveness of Trichogramma pretiosum in controlling lepidopterous pests of soybean crops. Agrociencia Uruguay, 24(NE2), Article e419. http://doi.org/10.31285/agro.24.419

Blackmer, J. L., Eiras, A. E., \& de Souza, C. L. M. (2001). Oviposition preference of Neoleucinodes elegantalis (Guenée) (Lepidoptera: Crambidae) and parasitism rates by Trichogramma pretiosum Riley (Hymenoptera: Trichogrammatidae) 
on Lycopersicon esculentum in São José de Ubá, RJ, Brazil. Neotropical Entomology, 30(1), 89-95. https://doi. org/10.1590/S1519-566X2001000100014

Cabello, T., Gallego Granados, J. R., Vila, E., Soler A., del Pino, M., Carnero, A., Hernández-Suárez, E., \& Polaszek, A. (2009). Biological control of the South American tomato pinworm, Tuta absoluta (Lep.: Gelechiidae), with releases of Trichogramma achaeae (Hym.: Trichogrammatidae) in tomato greenhouses of Spain. IOBC/WPRS Bulletin, 49, 225-230.

Cabello, T., Gámez, M., Varga, Z., Garay, J., Carreño, R., Gallego, J. R., Fernández, F. J., \& Vila, E. (2012). Selection of Trichogramma spp. (Hym.: Trichogrammatidae) for biological control of Tuta absoluta (Lep.: Gelechiidae) in greenhouse by entomo-ecological simulation model. IOBC/WPRS Bulletin, 80, 171-176.

Cabezas G., F., Melo, M., García, M. S., Diez-Rodríguez, G. I., \& Nava, D. E. (2013). Parasitismo de Trichogramma pretiousum (Hymenoptera: Trichogrammatidae) sobre Spodoptera cosmioides (Lepidoptera: Noctuidae) a diferentes temperaturas. Revista Colombiana de Entomología, 39(2), 216-220.

Chamaani, N., \& Poorjavad, N. (2020). Low efficiency of four indigenous Trichogramma wasp populations, collected from tomato crops, in controlling the invasive pest Tuta absoluta in Iran. Bulletin of Insectology, 73(2), 171-180.

Díaz, M., A. E., \& Brochero, H. L. (2012). Parasitoides asociados al perforador del fruto de las solanáceas Neoleucinodes elegantalis (Lepidoptera: Crambidae) en Colombia. Revista Colombiana de Entomología, 38(1), 50-57.

Di Rienzo, J. A., Casanoves, F., Balzarini, M. G., González, L., Tablada, M., \& Robledo C. W. (2020). InfoStat versión 2020. Universidad Nacional de Córdoba. http://www.infostat.com.ar

Fernandes, M. G., Busoli, A. C., \& Degrande, P. E. (1999). Natural parasitism of Alabama argillacea Hub. and Heliothis virescens Fab. by Trichogramma pretiosum Riley (Hym: Trichogrammatidae) on cotton in the state of Mato Grosso do Sul. Anais da Sociedade Entomológica do Brasil, 28(4), 695-701 . https://doi.org/10.1590/S0301-80591999000400012

Ferrán-García, M., \& Vercher, R. (2010). Descripción, origen y expansión de Tuta absoluta (Lepidoptera: Gelechiidae). Phytoma, 21, 16-20.

Fuentes, S., F., Ferrer, W. F., \& Salas A. J. (2012). Reseña histórica del control biológico en Centroamérica y el Caribe. Editorial Académica Española.

Gaffar, S. A., Mikhail, W. Z., Sobbhy, H., Elmasry, A. T., \& Omar, H. I. H. (2016). Integrated pest management of leafminer moth, Tuta absoluta on tomato plants. Egyptian Academy Journal of Biological Science, Toxicology \& Pest Control, 8(1), 43-55. https://doi.org/10.21608/EAJBSF.2016.17132

Gallego, J. R., Mellado-López, L., \& Cabello, T. (2020). Selección de una especie de Trichogramma (Hym., Trichogrammatidae) para el control biológico de la polilla de la patata Phthorimaea operculella (Lep., Gelechiidae) mediante el estudio del comportamiento de parasitación del huésped. Información Técnica Económica Agraria, 116(1), 2-18. https://doi. org/10.12706/itea.2019.013

Laurentis, V. L., Gomes Ramalho, D., Alves Santos, N., Pereira Carvalho, V. F., Vacari, A. M., De Bortoli, S. A., Sola Veneziani, R. C., da Costa Inácio, G., \& Gomes Dami, B. (2019). Performance of Trichogramma pretiosum Riley (Hymenoptera: Trichogrammatidae) on eggs of Helicoverpa armigera (Hübner) (Lepidoptera: Noctuidae). Scientific Reports, 9 , Article 1156. https://doi.org/10.1038/s41598-018-37797-9

Mansour, R., Cherif, A., Attia-Barhoumi, S., Zappala, L., \& Grissa-Lebdi, K. (2019). Tuta absoluta in Tunisia: ten years of invasion and management. Phytoparasitica, 47, 461-474. https://doi.org/10.1007/s12600-019-00748-9

Agron. Mesoam. 33(1): 43350, 2022

ISSN 2215-3608 doi:10.15517/am.v33i2.43350 
Massaroli, A., Butnariu, A.R., \& Doetzer, A.K. (2014). Occurrence of Trichogramma Parasitoids in Eggs of Soybean Lepidopteran Pests in Mato Grosso, Brazil. International Journal of Biology, 6(2), 97-103. http://doi.org/10.5539/ ijb.v6n2p97

Morales, J., Vásquez, C., Pérez B., N. L., Valera, N., Ríos, Y., Arrieche, N., \& Querino, R. B. (2007). Especies de Trichogramma (Hymenoptera: Trichogrammatidae) parasitoides de huevos de Lepidópteros en el Estado Lara, Venezuela. Neotropical Entomology, 36(4), 542-546. https://doi.org/10.1590/S1519-566X2007000400011

Moura de Oliveira, C., Vargas de Oliveira, J., Silva Barbosa, D. R., Oliveira Breda, M., de Franca, S. M., \& Ribeiro Duarte, B. L. (2017). Biological parameters and thermal requirements of T. pretiosum for the management of tomato fruit borer (Lepidoptera: Crambidae) in tomatoes. Crop Protection, 99, 39-44. https://doi.org/10.1016/j.cropro.2017.04.005

Mukwa, L. F. T., Mukendi, J., Adakate, F. G., Bugeme, D. M., Kalonji-Mbuyi, A., \& Ghimire, S. (2021). First report of the South American tomato pinworm Tuta absoluta (Meyrick) (Lepidoptera: Gelechiidae) and its damage in the Democratic Republic of Congo. BioInvasions Records, 10(1), 33-44. https://doi.org/10.3391/bir.2021.10.1.05

Riquelme Virgala, M. B., \& Botto, E. N. (2010). Biological studies on Trichogrammatoidea bactrae Nagaraja egg parasitoid of Tuta absoluta Meyrick (Lepidoptera: Gelechiidae). Neotropical Entomology, 39(4), 612-617. https://doi.org/10.1590/ S1519-566X2010000400023

Rodrigues Gonçalves-Gervásio, R. C., Iorio Ciociola, A., Costa Santa-Cecília, L. V., \& Maluf, W. R. (2000). Egg parasitism of Tuta absoluta by Trichogramma pretiosum in different genotypes of tomato. Pesquisa Agropecuária Brasileira, 35(6), 1269-1274. https://doi.org/10.1590/S0100-204X2000000600024

Saeidi, Z., \& Raeesi, M. (2021). Integration of resistant variety and biological agent to control tomato leaf miner, Tuta absoluta (Meyrick), under greenhouse conditions. Bulletin of Entomological Research, 111(3), 357-363. https://doi. org/10.1017/S0007485320000735

Salas, J., Álvarez, C., \& Parra, A. (1991). Contribución al conocimiento de la ecología del perforador del fruto de tomate, Neoleucinodes elegantalis (Lepidoptera: Pyraustidae). Agronomía Tropical, 41(5-6), 275-283.

Sarhan,A. A., Osman, M. A. M., Mandour, N. S., \& Abd El-Hady, M. A. (2015). Parasitization capability of four Trichogrammatid species against the tomato leaf miner, Tuta absoluta (Meyrick) (Lepidoptera: Gelechiidae) under different releasing regimes. Egyptian Journal of Biological Pest Control, 25(1), 107-112.

Shäfer, L., \& Herz, A. (2020). Suitability of European Trichogramma species as biocontrol agents against the tomato leaf miner Tuta absoluta. Insects, 11(6), 357.

Silva Alarcón, I. (2020). Evaluación del control biológico, etológico y químico de Neoleucinodes elegantalis en tomate (Lycopersicon esculentum). Revista Ambientellanía, 3(1), 31-40. http://revistas.unellez.edu.ve/index.php/ ambientellania/article/view/888

Tarusikirwa, V. S., Mutamiswa, R., Machekano, H., Chidawanyika, F., \& Nyamukondiwa, C. (2020). Tuta absoluta (Meyrick) (Lepidoptera: Gelechiidae) on the "Offensive" in Africa: Prospects for Integrated Management Initiatives. Insects, 11(11), Article 764. https://doi.org/10.3390/insects11110764

Zouba, A., \& Mahjoubi, K. (2010). Biological control of Tuta absoluta (Lepidoptera: Gelechiidae) with release of Trichogramma cacoeciae (Hymenoptera: Trichogrammatidae) in tomato greenhouses in Tunisia. The African Journal of Plant Science and Biotechnology, 4(Special Issue 2), 85-87.

Agron. Mesoam. 33(1): Artículo 43350, 2022 ISSN 2215-3608 doi:10.15517/am.v33i2.43350 